Göteborg ITP 97-03

April 1997

hep-th/9704209

(Appendix B inserted May 1997 and a new introduction August 1997)

\title{
Antisymplectic gauge theories
}

\author{
Igor Batalin [ and Robert Marnelius [2 \\ Institute of Theoretical Physics \\ Chalmers University of Technology \\ S-412 96 Göteborg, Sweden
}

\begin{abstract}
A general field-antifield BV formalism for antisymplectic first class constraints is proposed. It is as general as the corresponding symplectic BFV-BRST formulation and it is demonstrated to be consistent with a previously proposed formalism for antisymplectic second class constraints through a generalized conversion to corresponding first class constraints. Thereby the basic concept of gauge symmetry is extended to apply to quite a new class of gauge theories potentially possible to exist.
\end{abstract}

\footnotetext{
${ }^{1}$ On leave of absence from P.N.Lebedev Physical Institute, 117924 Moscow, Russia E-mail: batalin@lpi.ac.ru

${ }^{2}$ E-mail: tferm@fy.chalmers.se
} 


\section{Introduction}

The field-antifield BV formalism [1] is a Lagrangian path integral method to quantize general gauge theories (important early contributions are [2]-[4]). It has been shown to work for an ever increasing number of models. In the BV formalism one introduces antifields with opposite Grassmann parities to all field and ghost variables. It involves in a crucial way also an antibracket operation and a nilpotent differential $\Delta$-operator. The understanding of the formalism was further deepened in [5]-[9] and in [10]-[14]. In the approach of the latter papers a coordinate invariant general covariant formulation was developed. The field-antifield variables are here considered as arbitrary coordinates on an antisymplectic manifold $\mathcal{M}$. (The standard BV formalism may then be viewed as formulated in terms of antisymplectic Darboux coordinates.) In this formalism the geometric coordinate invariant properties is formally demonstrated. Furthermore, the formalism specifies the conditions a consistent invariant measure density has to satisfy. It involves also new ingredients like a hypergauge formulation [10, 14] and a multilevel formalism [11]. Gauge invariance is demonstrated in general terms. Among the further generalizations are deformed $\Delta$-operators in [12] and an $\operatorname{Sp}(2)$ version in [14, 15]. In [10] it was also shown how antisymplectic second class constraints may be introduced and treated consistently within this formalism. In this paper we continue this set of formal generalizations with still another one. Here we show that the path integral may be formulated on a large antisymplectic manifold also in the presence of antisymplectic first class constraints. All required conditions are shown to be formally satisfied. This is therefore a major further generalization of the general covariant BV formalism. The beautiful general mathematical structure of the BV formalism is thereby further extended. However, it remains to demonstrate the existence of examples which satisfy the generalizations suggested by the obtained formal results. Although we expect them to exist this is certainly a nontrivial issue. Anyway the formal results suggest alternative formulations which could turn out to be useful. Particularly the results of the present paper could allow for formulations with specific global symmetries which are preferable for some reasons. At a more speculative level our results show the existence of new types of gauge theories in an antisymplectic quantum theory in the spirit of [5]-[8].

In section 2 we recapitulate some basic properties of the general covariant BV formalism. In section 3 and appendices $\mathrm{A}$ and $\mathrm{B}$ we present our formulation and its formal properties. In section 4 and appendix $\mathrm{C}$ we consider then a generalized conversion of antisymplectic second class constraints into corresponding first class ones by means of an extension of the field-antifield manifold $\mathcal{M}$. This provides for an explicit formal verification of the formalism. Throughout the paper we make use of deWitt's compact notation which reduces the treatment to a finite dimensional one. In principle all functionals may be either local or nonlocal.

\section{Basics of general covariant BV formalism}

The basic object in field-antifield quantization is the nilpotent fermionic second order differential operator

$$
\Delta \equiv \frac{1}{2}(-1)^{\varepsilon_{A}} \rho^{-1} \partial_{A} \rho E^{A B} \partial_{B}
$$


where $\partial_{A}$ are derivatives with respect to local coordinates $\Gamma^{A}, A=1, \ldots, 2 N$, on an antisymplectic manifold $\mathcal{M}$. Their Grassmann parities are $\varepsilon\left(\Gamma^{A}\right) \equiv \varepsilon_{A} \in\{0,1\} .\left(\Gamma^{A}\right.$ are generalized fields and antifields.) $\rho(\Gamma)$ is a measure density and $E^{A B}$ an odd metric tensor with the properties: $E^{A B}=E^{B A}(-1)^{\varepsilon_{A}+\varepsilon_{B}+\varepsilon_{A} \varepsilon_{B}}$ and $\varepsilon\left(E^{A B}\right)=\varepsilon_{A}+\varepsilon_{B}+1$. Another basic object in the field-antifield formalism is the antibracket given by

$$
(F, G) \equiv(-1)^{\varepsilon_{F}} \Delta(F G)-(-1)^{\varepsilon_{F}}(\Delta F) G-F \Delta G=F \overleftarrow{\partial}_{A} E^{A B} \partial_{B} G
$$

for arbitrary functions $F, G$ on $\mathcal{M}$. It satisfies

$$
\begin{aligned}
& \varepsilon((F, G))=\varepsilon_{F}+\varepsilon_{G}+1, \quad(F, G)=-(G, F)(-1)^{\left(\varepsilon_{F}+1\right)\left(\varepsilon_{G}+1\right)}, \\
& (F, G H)=(F, G) H+(-1)^{\varepsilon_{G}\left(\varepsilon_{F}+1\right)} G(F, H), \\
& ((F, G), H)(-1)^{\left(\varepsilon_{F}+1\right)\left(\varepsilon_{H}+1\right)}+\operatorname{cycle}(F, G, H) \equiv 0, \\
& \Delta(F, G)=(\Delta F, G)+(-1)^{\left(\varepsilon_{F}+1\right)}(F, \Delta G) .
\end{aligned}
$$

The measure density $\rho$ satisfies also

$$
(-1)^{\left(\varepsilon_{A}+\varepsilon_{C}\right)} \rho^{-1} \partial_{A} \rho E^{A B} \partial_{B} \rho^{-1} \partial_{C} \rho E^{C D}=0 .
$$

All these properties follow from the nilpotency of the $\Delta$-operator (11), i.e. $\Delta^{2}=0$.

On $\mathcal{M}$ we assume the existence of a quantum master action $W$ satisfying

$$
\Delta e^{\frac{i}{\hbar} W}=0 \Leftrightarrow \frac{1}{2}(W, W)=i \hbar \Delta W
$$

In terms of $W$ there is another nilpotent second order differential operator $\sigma_{W}$ defined by 6]

$$
\sigma_{W} F \equiv \frac{\hbar}{i} e^{-\frac{i}{\hbar} W} \Delta e^{\frac{i}{\hbar} W} F=(W, F)-i \hbar \Delta F
$$

which satisfies $\sigma_{W}^{2}=0$ and

$$
\begin{aligned}
& \sigma_{W}(F, G)=\left(\sigma_{W} F, G\right)+(-1)^{\varepsilon_{F}+1}\left(F, \sigma_{W} G\right), \\
& \sigma_{W} F G=\left(\sigma_{W} F\right) G+(-1)^{\varepsilon_{F}} F\left(\sigma_{W} G\right)-i \hbar(-1)^{\varepsilon_{F}}(F, G) .
\end{aligned}
$$

Given two solutions $W$ and $X$ of the master equation (5), one has

$$
\left[\sigma_{W}, \sigma_{X}\right] F=-\frac{1}{2}((-W+X,-W+X), F) .
$$

The path integral in this generalized BV formalism is given by

$$
Z=\int \exp \left\{\frac{i}{\hbar}[W+X]\right\} \rho[d \Gamma][d \eta],
$$

where $W$ is the above quantum master action and $X$ a hyper gauge-fixing master action which also satisfies the quantum master equation (5). $\eta^{a}$ are second level Lagrange multipliers [12, 13] with no corresponding antifields. This means that $\mathcal{M}$ is viewed as containing first level Lagrange multipliers $\lambda^{a}$ and their antifields $\lambda_{a}^{*}\left(\left\{\Gamma^{A}\right\}=\left\{\Gamma_{0}^{A}, \lambda^{a}, \lambda_{a}^{*}\right\}\right)$ with the Grassmann parities $\varepsilon\left(\eta^{a}\right)=\varepsilon\left(\lambda_{a}^{*}\right)=\varepsilon\left(\lambda^{a}\right)+1$. The actions $W$ and $X$ have then the form

$$
W=W_{0}\left(\Gamma_{0}\right)+\lambda_{a}^{*} \eta^{a}, \quad X=G_{a}\left(\Gamma_{0}\right) \lambda^{a}+\ldots,
$$


where $G_{a}$ are hyperconstraints that fixes the antifields in $\left\{\Gamma_{0}^{A}\right\}$. Also $W_{0}$ satisfies the master equation (5). Under these conditions one may show that the path integral (9) is independent of the precise form of the hypergauge conditions $G_{a}$ (X-independence) [14].

If we introduce some constraints $\Theta^{\alpha}=0, \alpha=1, \ldots, 2 n<2 N$, on $\mathcal{M}$ such that $E^{\alpha \beta} \equiv\left(\Theta^{\alpha}, \Theta^{\beta}\right)$ is invertible, then we may define a "Dirac" antibracket by the expression [10]

$$
(F, G)_{(\mathcal{D})} \equiv(F, G)-\left(F, \Theta^{\alpha}\right) E_{\alpha \beta}\left(\Theta^{\beta}, G\right),
$$

where $E_{\alpha \beta}$ is the invers to $E^{\alpha \beta}$. Obviously

$$
(F, G)_{(\mathcal{D})}=F \overleftarrow{\partial}_{A} E_{(\mathcal{D})}^{A B} \partial_{B} G, \quad E_{(\mathcal{D})}^{A B} \equiv E^{A B}-E^{A C}\left(\partial_{C} \Theta^{\alpha}\right) E_{\alpha \beta}\left(\Theta^{\beta} \overleftarrow{\partial}_{D}\right) E^{D B}
$$

Since $\left(F, \Theta^{\alpha}\right)_{(\mathcal{D})}=0$ for any $F$ the metric $E_{(\mathcal{D})}^{A B}$ is degenerate on $\mathcal{M}$. However, even in terms of such a metric there is a consistent path integral and it is given by [10]

$$
Z_{(\mathcal{D})}=\int \exp \left\{\frac{i}{\hbar}[W+X]\right\} \prod_{\alpha} \delta\left(\Theta^{\alpha}\right) \rho_{(\mathcal{D})}[d \Gamma][d \eta]
$$

where now $W$ and $X$ satisfy the quantum master equations (5) with $\Delta$ replaced by

$$
\Delta_{(\mathcal{D})} \equiv \frac{1}{2}(-1)^{\varepsilon_{A}} \rho_{(\mathcal{D})}^{-1} \partial_{A} \rho_{(\mathcal{D})} E_{(\mathcal{D})}^{A B} \partial_{B}
$$

Thus, for antisymplectic second class constraints $\Theta^{\alpha}=0$ there is a consistent formulation already. We shall now propose a consistent formulation for corresponding first class constraints.

\section{Field-antifield formalism with first class constraints}

Let us call $T_{\alpha}=0$ antisymplectic first class constraints provided $T_{\alpha}$ satisfy

$$
\left(T_{\alpha}, T_{\beta}\right)=T_{\gamma} U_{\alpha \beta}^{\gamma}
$$

In the presence of such constraints we propose the following path integral

$$
\begin{aligned}
Z_{T} & =\int \exp \left\{\frac{i}{\hbar}[W+X]\right\} \prod_{\alpha} \delta\left(T_{\alpha}\right) \prod_{\beta} \delta\left(\chi^{\beta}\right) \frac{1}{\operatorname{sdet}\left(\chi^{\gamma}, T_{\delta}\right)} \rho(\Gamma)[d \Gamma][d \eta]= \\
& =\int \exp \left\{\frac{i}{\hbar}\left[W+X+\overline{\mathcal{C}}_{\alpha}\left(\chi^{\alpha}, T_{\beta}\right) \mathcal{C}^{\beta}+T_{\alpha} \pi^{\alpha}+\xi_{\alpha} \chi^{\alpha}\right]\right\} d \mu, \\
d \mu & \equiv \rho(\Gamma)[d \Gamma][d \eta][d \pi][d \xi][d \mathcal{C}][d \overline{\mathcal{C}}],
\end{aligned}
$$

where $\rho(\Gamma)$ is a gauge independent measure density, and where $\chi^{\alpha}=0$ are gauge-fixing conditions to $T_{\alpha}=0$, i.e. $\left(\chi^{\gamma}, T_{\delta}\right)$ is required to be invertible. The Grassmann parities of the field variables in (16) are

$$
\varepsilon\left(\pi^{\alpha}\right)=\varepsilon\left(\mathcal{C}^{\alpha}\right)=\varepsilon\left(\overline{\mathcal{C}}_{\alpha}\right)=\varepsilon_{\alpha} \equiv \varepsilon\left(T_{\alpha}\right), \quad \varepsilon\left(\xi_{\alpha}\right)=\varepsilon\left(\chi^{\alpha}\right)=\varepsilon_{\alpha}+1 .
$$

The first class constraints $T_{\alpha}$ are in addition to (15) required to satisfy

$$
\begin{aligned}
& \sigma_{W} T_{\alpha}=T_{\beta} P_{\alpha}^{\beta}-i \hbar U_{\beta \alpha}^{\beta}(-1)^{\varepsilon_{\beta}}, \\
& \sigma_{X} T_{\alpha}=T_{\beta} Q_{\alpha}^{\beta}-i \hbar U_{\beta \alpha}^{\beta}(-1)^{\varepsilon_{\beta}},
\end{aligned}
$$


which also may be viewed as conditions on $W$ and $X$. A general representation of the path integral (16) is given in appendix $\mathrm{A}$.

The path integral $Z_{T}$ is invariant under the supertransformation

$$
\delta \Gamma^{A}=\left(\Gamma^{A}, T_{\alpha}\right) \mathcal{C}^{\alpha} \mu
$$

where $\mu$ is an odd constant. It leads to

$$
\begin{aligned}
& \delta T_{\alpha}=T_{\gamma} U_{\alpha \beta}^{\gamma} \mathcal{C}^{\beta} \mu, \quad \delta \chi^{\alpha}=\left(\chi^{\alpha}, T_{\beta}\right) \mathcal{C}^{\beta} \mu \\
& \delta\left(\overline{\mathcal{C}}_{\alpha}\left(\chi^{\alpha}, T_{\beta}\right) \mathcal{C}^{\beta}\right)=\frac{1}{2} \overline{\mathcal{C}}_{\alpha}\left(\chi^{\alpha}, T_{\delta}\right) U_{\beta \gamma}^{\delta} \mathcal{C}^{\gamma} \mathcal{C}^{\beta}(-1)^{\varepsilon_{\beta}}+\frac{1}{2} T_{\delta} \overline{\mathcal{C}}_{\alpha}\left(\chi^{\alpha}, U_{\beta \gamma}^{\delta}\right) \mathcal{C}^{\gamma} \mathcal{C}^{\beta}(-1)^{\varepsilon_{\beta}}(20)
\end{aligned}
$$

and

$$
\delta(W+X)=2 i \hbar\left(\Delta T_{\alpha}-U_{\beta \alpha}^{\beta}(-1)^{\varepsilon_{\beta}}\right) \mathcal{C}^{\alpha} \mu+T_{\beta}\left(P_{\alpha}^{\beta}+Q_{\alpha}^{\beta}\right) \mathcal{C}^{\alpha} \mu
$$

from (18). Furthermore, it gives rise to the following Jacobian

$$
J=1+2\left(\Delta T_{\alpha}\right) \mathcal{C}^{\alpha} \mu
$$

All these contributions from the transformation (19) in the integrand of $Z_{T}$ are compensated by the transformations

$$
\begin{aligned}
\delta \mathcal{C}^{\delta}= & -\frac{1}{2} U_{\beta \gamma}^{\delta} \mathcal{C}^{\gamma} \mathcal{C}^{\beta}(-1)^{\varepsilon_{\beta}} \mu \\
\delta \pi^{\delta}= & -\frac{1}{2} \overline{\mathcal{C}}_{\alpha}\left(\chi^{\alpha}, U_{\beta \gamma}^{\delta}\right) \mathcal{C}^{\gamma} \mathcal{C}^{\beta}(-1)^{\varepsilon_{\beta}} \mu- \\
& -U_{\alpha \beta}^{\delta} \mathcal{C}^{\beta} \pi^{\alpha}(-1)^{\varepsilon_{\alpha}} \mu-\left(P_{\alpha}^{\delta}+Q_{\alpha}^{\delta}\right) \mathcal{C}^{\alpha} \mu \\
\delta \overline{\mathcal{C}}_{\alpha}= & \mu \xi_{\alpha},
\end{aligned}
$$

together with the resulting contributions from the corresponding Jacobians

$$
\delta \mathcal{C}^{\delta} \frac{\overleftarrow{\partial}}{\partial \mathcal{C}^{\delta}}(-1)^{\varepsilon_{\delta}}=-U_{\beta \gamma}^{\beta} \mathcal{C}^{\gamma}(-1)^{\varepsilon_{\beta}} \mu, \quad \delta \pi^{\delta} \frac{\overleftarrow{\partial}}{\partial \pi^{\delta}}(-1)^{\varepsilon_{\delta}}=-U_{\beta \gamma}^{\beta} \mathcal{C}^{\gamma}(-1)^{\varepsilon_{\beta}} \mu
$$

The path integral $Z_{T}$ is also independent of the gauge-fixing functions $\chi^{\alpha}$. To see this consider the shift

$$
\chi^{\alpha} \rightarrow \chi^{\alpha}+\delta \chi^{\alpha}
$$

in $Z_{T}$. It is in fact exactly compensated by the transformation (19) with the choice

$$
\mu=\frac{i}{\hbar} \overline{\mathcal{C}}_{\alpha} \delta \chi^{\alpha}
$$

since when compared to the previous transformation with $\mu$ constant this choice gives rise to the following additional contribution to the Jacobian

$$
\begin{aligned}
\delta J & \equiv\left(\Gamma^{A}, T_{\alpha}\right) \mathcal{C}^{\alpha} \frac{i}{\hbar}\left(\overline{\mathcal{C}}_{\beta} \delta \chi^{\beta} \overleftarrow{\partial}_{A}\right)(-1)^{\varepsilon_{A}}+\frac{i}{\hbar}(-1)^{\varepsilon_{\alpha}} \frac{\partial}{\partial \overline{\mathcal{C}}_{\alpha}}\left(\overline{\mathcal{C}}_{\beta} \delta \chi^{\beta}\right) \xi_{\alpha}= \\
& =-\frac{i}{\hbar} \overline{\mathcal{C}}_{\beta}\left(\delta \chi^{\beta}, T_{\alpha}\right) \mathcal{C}^{\alpha}-\frac{i}{\hbar} \xi_{\alpha} \delta \chi^{\alpha} .
\end{aligned}
$$




\section{Conversion and the Abelian case}

We shall now apply and verify the general formulation above. We consider then a generalized conversion of antisymplectic second class constraints into corresponding first class ones. Within the ordinary Hamiltonian formalism the conversion mechanism has been formulated in general terms in [16, 17] (see also [18]). It has been applied to many models. One interesting application is the new approach to geometric quantization in [19] which is mainly based on [17. In the following application to the field-antifield formalism a new ingredient appears since we not only have antibrackets, which corresponds to Poisson brackets, but also the nilpotent differential $\Delta$-operator.

Consider the second class constraints $\Theta^{\alpha}=0, \alpha=1, \ldots, 2 n<2 N$, on $\mathcal{M}$ which by definition are such that $E^{\alpha \beta} \equiv\left(\Theta^{\alpha}, \Theta^{\beta}\right)$ is invertible. We now convert these constraints into abelian first class constraints by extending the original antisymplectic manifold $\mathcal{M}$. Introduce therefore the additional field-antifield coordinates $\Phi^{\alpha}$ with the Grassmann parities $\varepsilon\left(\Phi^{\alpha}\right)=\varepsilon\left(\Theta^{\alpha}\right)=\varepsilon_{\alpha}$. On the resulting extended manifold, $\mathcal{M}_{\text {ext }} \equiv \mathcal{M} \oplus\left\{\Phi^{\alpha}\right\}$, we define then an extended antibracket with the extended metric

$$
\left(\Gamma^{A}, \Gamma^{B}\right)_{\mathrm{ext}}=E^{A B}, \quad\left(\Gamma^{A}, \Phi^{\alpha}\right)_{\mathrm{ext}}=0, \quad\left(\Phi^{\alpha}, \Phi^{\beta}\right)_{\mathrm{ext}}=\omega^{\alpha \beta},
$$

where $\omega^{\alpha \beta}$ is an odd invertible constant matrix. On $\mathcal{M}_{\text {ext }}$ we may then define first class constraints $T^{\alpha}$ satisfying

$$
\left(T^{\alpha}, T^{\beta}\right)_{\mathrm{ext}}=0,\left.\quad T^{\alpha}\right|_{\Phi=0}=\Theta^{\alpha} .
$$

These functions may be constructed perturbatively with the ansatz

$$
T^{\alpha}(\Gamma, \Phi)=\Theta^{\alpha}(\Gamma)+\sum_{n=1}^{\infty} \frac{1}{n !} \Phi^{\beta_{n}} \cdots \Phi^{\beta_{1}} X_{\beta_{1} \cdots \beta_{n}}^{\alpha}(\Gamma) .
$$

We may furthermore construct gauge invariant functions $\bar{G}(\Gamma, \Phi)$ to any function $G(\Gamma)$ by the conditions

$$
\left(\bar{G}, T^{\alpha}\right)_{\mathrm{ext}}=0,\left.\quad \bar{G}\right|_{\Phi=0}=G .
$$

Also these conditions may be solved perturbatively with an ansatz of the form

$$
\bar{G}(\Gamma, \Phi)=G(\Gamma)+\sum_{n=1}^{\infty} \frac{1}{n !} \Phi^{\beta_{n}} \cdots \Phi^{\beta_{1}} Y_{\beta_{1} \cdots \beta_{n}}(\Gamma) .
$$

In appendix B it is shown that

$$
\left.(\bar{F}, \bar{G})_{\mathrm{ext}}\right|_{\Phi=0}=(F, G)_{(\mathcal{D})},
$$

where the right-hand side is the Dirac antibracket (11). One may also show that (see below)

$$
\left.\Delta_{\text {ext }} \bar{G}\right|_{\Phi=0}=\Delta_{(\mathcal{D})} G
$$

provided

$$
\Delta_{\text {ext }} T^{\alpha}=0
$$


$\Delta_{\text {ext }}$ is the corresponding $\Delta$-operator to (1) on the extended manifold $\mathcal{M}_{\text {ext }}$ with the metric (28) and with a measure density $\rho(\Gamma, \Phi)$ satisfying (35). To show (33) it is sufficient to solve (30) and (32) up to the first order in $\Phi$ as is shown in appendix B, while (34) requires a solution up to second order.

The gauge independent path integral (16) is here given by

$$
Z_{T}=\int \exp \left\{\frac{i}{\hbar}\left[W_{\mathrm{ext}}+X_{\mathrm{ext}}\right]\right\} \prod_{\alpha} \delta\left(T^{\alpha}\right) \prod_{\beta} \delta\left(\chi_{\beta}\right) \frac{1}{\operatorname{sdet}\left(\chi_{\gamma}, T^{\delta}\right)} \rho(\Gamma, \Phi)[d \Gamma][d \Phi][d \eta],
$$

where $W_{\text {ext }}$ and $X_{\text {ext }}$ satisfy the quantum master equations

$$
\Delta_{\text {ext }} e^{\frac{i}{\hbar} W_{\text {ext }}}=0, \quad \Delta_{\text {ext }} e^{\frac{i}{\hbar} X_{\text {ext }}}=0 .
$$

According to (18) we have additional conditions like (see appendix A)

$$
\left(W_{\mathrm{ext}}, T^{\alpha}\right)_{\mathrm{ext}}=i \hbar \Delta_{\mathrm{ext}} T^{\alpha}, \quad\left(X_{\mathrm{ext}}, T^{\alpha}\right)_{\mathrm{ext}}=i \hbar \Delta_{\mathrm{ext}} T^{\alpha} .
$$

They are easy to solve for measure densities satisfying (35) since these conditions then reduce to

$$
\left(W_{\text {ext }}, T^{\alpha}\right)_{\mathrm{ext}}=\left(X_{\mathrm{ext}}, T^{\alpha}\right)_{\mathrm{ext}}=0 .
$$

This implies that $W_{\text {ext }}$ and $X_{\text {ext }}$ in this case are gauge invariant extensions of $W$ and $X$ defined by

$$
\left.W(\Gamma) \equiv W_{\text {ext }}\right|_{\Phi=0},\left.\quad X(\Gamma) \equiv X_{\text {ext }}\right|_{\Phi=0},
$$

which means that $W_{\text {ext }}=\bar{W}$ and $X_{\text {ext }}=\bar{X}$. It follows now that the path integral (36) in e.g. the gauge $\chi_{\alpha}=\omega_{\alpha \beta} \Phi^{\beta}$ reduces to

$$
Z_{T}=\int \exp \left\{\frac{i}{\hbar}[W+X]\right\} \prod_{\alpha} \delta\left(\Theta^{\alpha}\right) \frac{1}{\operatorname{sdet}\left(X_{\gamma}^{\delta}\right)} \rho(\Gamma, 0)[d \Gamma][d \eta],
$$

which when compared to the second class expression $Z_{(\mathcal{D})}$ in $(13)$ requires the boundary condition

$$
\rho(\Gamma, 0)=\rho_{(\mathcal{D})}(\Gamma) \operatorname{sdet}\left(X_{\gamma}^{\delta}\right)
$$

where $X_{\gamma}^{\delta}$ is the first order coefficient in the expansion (30). That $W$ and $X$ satisfy the appropriate master equations follows from (33) and (34).

Another equivalent but more explicit and transparent way to derive the equivalence between (13) and (36) is to first construct gauge invariant coordinates $\bar{\Gamma}^{A}(\Gamma, \Phi)$ defined by

$$
\left(\bar{\Gamma}^{A}, T^{\alpha}\right)_{\mathrm{ext}}=0,\left.\quad \bar{\Gamma}^{A}\right|_{\Phi=0}=\Gamma^{A}
$$

which also may be solved by a perturbative ansatz like (32). Then we have $\bar{G}=G(\bar{\Gamma})$ for any gauge invariant function and

$$
\left.\left(\bar{\Gamma}^{A}, \bar{\Gamma}^{B}\right)_{\operatorname{ext}}\right|_{\Phi=0}=E_{(\mathcal{D})}^{A B} .
$$


Thus, the gauge invariant functions lives on the submanifold of $\mathcal{M}_{\text {ext }}$ spanned by $\bar{\Gamma}^{A}$. The same is true for the $\Delta$-operator as will be shown below.

It is convenient to change coordinates on $\mathcal{M}_{\text {ext }}$ from $\{\Gamma, \Phi\}$ to $\{\bar{\Gamma}, \Phi\}$. In terms of these coordinates we have

$$
\begin{aligned}
& (\bar{F}, \bar{G})_{\mathrm{ext}}=F(\bar{\Gamma}) \overleftarrow{\bar{\partial}}_{A} \bar{E}^{A B} \bar{\partial}_{B} G(\bar{\Gamma}) \\
& \bar{E}^{A B} \equiv\left(\bar{\Gamma}^{A}, \bar{\Gamma}^{B}\right)_{\mathrm{ext}}
\end{aligned}
$$

where $\bar{\partial}_{A}$ are derivatives with respect to $\bar{\Gamma}^{A}$. Furthermore, we have

$$
\begin{aligned}
& \Delta_{\text {ext }}=\frac{1}{2}(-1)^{\varepsilon_{A}} \bar{\rho}^{-1} \bar{\partial}_{A} \bar{\rho} \bar{E}^{A B} \bar{\partial}_{B}+\frac{1}{2}(-1)^{\varepsilon_{A}} \bar{\rho}^{-1} \bar{\partial}_{A} \bar{\rho}^{A \beta} \bar{\partial}_{\beta}+ \\
& +\frac{1}{2}(-1)^{\varepsilon_{\alpha}} \bar{\rho}^{-1} \bar{\partial}_{\alpha} \bar{\rho} \bar{E}^{\alpha B} \bar{\partial}_{B}+\frac{1}{2}(-1)^{\varepsilon_{\alpha}} \bar{\rho}^{-1} \bar{\partial}_{\alpha} \bar{\rho} \bar{E}^{\alpha \beta} \bar{\partial}_{\beta},
\end{aligned}
$$

where $\bar{\partial}_{\alpha}$ are derivatives with respect to $\Phi^{\alpha}$ while keeping $\bar{\Gamma}^{A}$ fixed, and where $\bar{\rho}$ is related to $\rho$ through the formula

$$
\bar{\rho}(\bar{\Gamma}, \Phi) \operatorname{sdet}\left(\partial_{A} \bar{\Gamma}^{B}\right)=\rho(\Gamma, \Phi) .
$$

The "bar"-metric in (46) is given in (45) and

$$
\begin{aligned}
& \bar{E}^{A \beta} \equiv\left(\bar{\Gamma}^{A}, \Phi^{\beta}\right)_{\mathrm{ext}}=\bar{\Gamma}^{A \overleftarrow{\partial}_{\alpha} \omega^{\alpha \beta},} \quad \bar{E}^{\alpha B} \equiv\left(\Phi^{\alpha}, \bar{\Gamma}^{B}\right)_{\mathrm{ext}}=\omega^{\alpha \beta} \partial_{\beta} \bar{\Gamma}^{B}, \\
& \bar{E}^{\alpha \beta} \equiv\left(\Phi^{\alpha}, \Phi^{\beta}\right)_{\mathrm{ext}}=\omega^{\alpha \beta} .
\end{aligned}
$$

Since the Jacobi identities yield

$$
\left(\bar{E}^{A B}, T^{\alpha}\right)_{\text {ext }}=0
$$

we have also

$$
\bar{E}^{A B}=E_{(\mathcal{D})}^{A B}(\bar{\Gamma})
$$

due to (44). The $\Delta$-operator expression (46) may be decomposed as follows

$$
\begin{aligned}
& \Delta_{\text {ext }}=\bar{\Delta}+K^{\alpha} \bar{\partial}_{\alpha}, \\
& \bar{\Delta}=\frac{1}{2}(-1)^{\varepsilon_{A}} \bar{\rho}^{-1} \bar{\partial}_{A} \bar{\rho} \bar{E}^{A B} \bar{\partial}_{B}+\frac{1}{2}(-1)^{\varepsilon_{\alpha}} \bar{\rho}^{-1}\left(\bar{\partial}_{\alpha} \bar{\rho} \bar{E}^{\alpha B}\right) \bar{\partial}_{B} .
\end{aligned}
$$

Obviously $\Delta_{\text {ext }} \bar{F}=\bar{\Delta} \bar{F}$ for any gauge invariant function $\bar{F}$. Furthermore, since the condition $\Delta_{\text {ext }} T^{\alpha} \equiv \Delta_{\text {ext }} \Theta^{\alpha}(\bar{\Gamma})=\bar{\Delta} \Theta^{\alpha}(\bar{\Gamma})=0$ implies that

$$
\frac{1}{2}(-1)^{\varepsilon_{\alpha}} \bar{\rho}^{-1}\left(\bar{\partial}_{\alpha} \bar{\rho} \bar{E}^{\alpha B}\right)=\frac{1}{2}(-1)^{\varepsilon_{A}} F_{A} \bar{E}^{A B}
$$

for any function $F_{A}$, we have

$$
\bar{\Delta}=\frac{1}{2}(-1)^{\varepsilon_{A}}\left(\bar{\partial}_{A}+F_{A}+\left(\bar{\partial}_{A} \ln \bar{\rho}\right)\right) \bar{E}^{A B} \bar{\partial}_{B} .
$$

Therefore, if we restrict $F_{A}$ such that $F_{A}+\left(\bar{\partial}_{A} \ln \bar{\rho}\right)$ only depends on $\bar{\Gamma}^{A}$ then also $\bar{\Delta}$ only depends on $\bar{\Gamma}^{A}$ due to (50). The nilpotency of $\Delta_{\text {ext }}$ requires then that $\bar{\Delta}$ is nilpotent which in turn implies 13]

$$
F_{A}+\left(\bar{\partial}_{A} \ln \bar{\rho}\right)=\bar{\partial}_{A} \ln \widetilde{\rho}(\bar{\Gamma})
$$


This result may equivalently be expressed as follows: In order for the measure density $\rho$ to satisfy (35) it should be such that

$$
\rho[d \Gamma][d \Phi]=\bar{\rho}[d \bar{\Gamma}][d \Phi]=\widetilde{\rho}(\bar{\Gamma})[d \bar{\Gamma}]\left[d T^{*}\right]
$$

for any measure density $\widetilde{\rho}(\bar{\Gamma})$ where $T_{\alpha}^{*}$ satisfies

$$
\left(T^{\alpha}, T_{\beta}^{*}\right)_{\mathrm{ext}}=\delta_{\beta}^{\alpha}, \quad\left(T_{\alpha}^{*}, T_{\beta}^{*}\right)_{\mathrm{ext}}=0, \quad \varepsilon\left(T_{\alpha}^{*}\right)=\varepsilon_{\alpha}+1 .
$$

We assert that there is a solution of the form

$$
T_{\alpha}^{*}(\Gamma, \Phi)=\Theta_{\alpha}^{*}(\Gamma)+\sum_{n=1}^{\infty} \frac{1}{n !} \Phi^{\beta_{n}} \cdots \Phi^{\beta_{1}} X_{\beta_{1} \cdots \beta_{n} \alpha}^{*}(\Gamma)
$$

where also the function $\Theta_{\alpha}^{*}(\Gamma)$ is to be determined (see appendix C). Obviously $\left.\Delta_{\text {ext }} \bar{G}\right|_{\Phi=0}=$ $\left.\bar{\Delta} \bar{G}\right|_{\Phi=0}=\Delta_{(\mathcal{D})} G$ in agreement with the assertion (34), provided

$$
\widetilde{\rho}(\bar{\Gamma})=\rho_{(\mathcal{D})}(\bar{\Gamma}),
$$

where $\rho_{(\mathcal{D})}$ is the Dirac measure density in (13) and (14). Thus, the $\Delta$-operator $\bar{\Delta}$ is just a gauge invariant extension of $\Delta_{(\mathcal{D})}$ on $\mathcal{M}_{\text {ext }}$. It should also be mentioned that the transformation (55) with the identification (58) is consistent with the boundary condition (42) due to the relation

$$
\left.\left(T_{\alpha}^{*}, \Phi^{\beta}\right)_{\operatorname{ext}}\right|_{\Phi=0, \Theta=0}=-\frac{1}{2}\left(X^{-1}\right)_{\alpha}^{\beta},
$$

which follows from (56) and (57) to lowest order in $\Phi^{\alpha}$ (see formula (C.9) in appendix C). 


\section{Acknowledgements}

I.A.B. thanks Klaus Bering and Poul Damgaard for stimulating discussions at an early stage of this work. I.A.B. would also like to thank Lars Brink for very warm hospitality at the Institute of Theoretical Physics, Chalmers and Göteborg University. The work is partially supported by grant INTAS-RFBR 95-0829. The work of I.A.B. is also supported by grants INTAS 93-2058, INTAS 93-0633, RFBR 96-01-00482, RFBR 96-02-17314, and NorFa 97.40.002-O.

\section{Appendix A}

\section{Invariant formulation of the path integral $Z_{T}$}

Let us extend the antisymplectic manifold $\mathcal{M}$ in section 2 by three sets of field-antifield pairs: $\left\{\mathcal{C}_{\alpha}^{*}, \mathcal{C}^{\alpha} ; \pi_{\alpha}^{*}, \pi^{\alpha} ; \overline{\mathcal{C}}_{\alpha}^{*}, \overline{\mathcal{C}}^{\alpha}\right\}$. Their Grassmann parities are

$$
\varepsilon\left(\mathcal{C}^{\alpha}\right)=\varepsilon\left(\pi^{\alpha}\right)=\varepsilon\left(\overline{\mathcal{C}}_{\alpha}^{*}\right)=\varepsilon_{\alpha} \equiv \varepsilon\left(T^{\alpha}\right), \quad \varepsilon\left(\mathcal{C}_{\alpha}^{*}\right)=\varepsilon\left(\pi_{\alpha}^{*}\right)=\varepsilon\left(\overline{\mathcal{C}}^{\alpha}\right)=\varepsilon_{\alpha}+1 .
$$

On this extended manifold, $\widetilde{\mathcal{M}}$, we define the bosonic charge

$$
\Omega=\left(T_{\alpha} \mathcal{C}^{\alpha}-\frac{1}{2} \mathcal{C}_{\alpha}^{*} U_{\beta \gamma}^{\alpha} \mathcal{C}^{\gamma} \mathcal{C}^{\beta}(-1)^{\varepsilon_{\beta}}+\ldots\right)-\pi_{\alpha}^{*} \overline{\mathcal{C}}^{\alpha}
$$

where $T_{\alpha}$ satisfies the algebra (15) and where the dots indicate terms independent of $\pi^{\alpha}$ and $\overline{\mathcal{C}}_{\alpha}^{*}$ such that $\Omega$ satisfies

$$
(\Omega, \Omega)=0,
$$

where the antibracket from now on is the extended one on $\widetilde{\mathcal{M}}$. An invariant path integral may then be written as

$$
Z_{T}=\int \exp \left\{\frac{i}{\hbar}[\widetilde{W}+\widetilde{X}+(\Psi, \Omega)]\right\} \widetilde{\rho}[d \Gamma][d \eta][d \mathcal{C}]\left[d \mathcal{C}^{*}\right][d \pi]\left[d \pi^{*}\right][d \overline{\mathcal{C}}]\left[d \overline{\mathcal{C}}^{*}\right],
$$

where $\widetilde{W}$ and $\widetilde{X}$ satisfy the extended master equations

$$
\widetilde{\Delta} e^{\frac{i}{\hbar} \widetilde{W}}=0, \quad \widetilde{\Delta} e^{\frac{i}{\hbar} \widetilde{X}}=0
$$

where in turn $\widetilde{\Delta}$ is the nilpotent $\Delta$-operator (何) extended to $\widetilde{\mathcal{M}}$ with $\widetilde{\rho}$. The objects $\Omega$, $\widetilde{W}$ and $\widetilde{X}$ are in addition required to satisfy

$$
\sigma_{\widetilde{W}} \Omega=\sigma_{\widetilde{X}} \Omega=0
$$

The gauge-fixing charge $\Psi$ is odd and is of the form

$$
\Psi=\overline{\mathcal{C}}_{\alpha}^{*} \chi^{\alpha}-\mathcal{C}_{\alpha}^{*} \pi^{\alpha}
$$

The solutions of (A.5) are

$$
\widetilde{W}=W(\Gamma)-\mathcal{C}_{\alpha}^{*} P_{\beta}^{\alpha} \mathcal{C}^{\beta}+\ldots, \quad \widetilde{X}=X(\Gamma)-\mathcal{C}_{\alpha}^{*} Q_{\beta}^{\alpha} \mathcal{C}^{\beta}+\ldots,
$$


The equations for $W$ and $X$ reduce to (5) if and only if $P_{\alpha}^{\alpha}=Q_{\alpha}^{\alpha}=0$. Otherwise $W$ and $X$ generalize to satisfy the modified equations with supertrace "anomalies" in their righthand sides (see appendix B). The formulation given in section 2 corresponds therefore to the first case. Notice that eq. (18) is obtained from (A.6) when (A.8) is inserted, and that with the choice (A.7) the path integral (A.4) reduces to (16) after the identifications $\pi_{\alpha}^{*} \equiv \xi_{\alpha}, \overline{\mathcal{C}}_{\alpha}^{*} \equiv \overline{\mathcal{C}}_{\alpha}$, and provided $\widetilde{\rho}=\rho(\Gamma)$ and $\chi^{\alpha}$ only depends on $\Gamma^{A}$.

The path integral (A.4) is invariant under the following transformation

$$
\delta \widetilde{\Gamma}^{\mathcal{A}}=\left(\widetilde{\Gamma}^{\mathcal{A}}, \Omega\right) \mu
$$

where $\widetilde{\Gamma}^{\mathcal{A}} \equiv\left\{\Gamma^{A} ; \mathcal{C}_{\alpha}^{*}, \mathcal{C}^{\alpha} ; \pi_{\alpha}^{*}, \pi^{\alpha} ; \overline{\mathcal{C}}_{\alpha}^{*}, \overline{\mathcal{C}}^{\alpha}\right\} \in \widetilde{\mathcal{M}}$ and where $\mu$ is an odd constant. The contribution to the Jacobian

$$
J-1=2(\widetilde{\Delta} \Omega) \mu
$$

is compensated by corresponding terms from (A.6). The path integral (A.4) is also independent of $\Psi$ since $\Psi \rightarrow \Psi+\delta \Psi$ is compensated by the additional contribution to the Jacobian from the transformation (A.9) with the choice $\mu=i \delta \Psi / \hbar$. Furthermore, (A.4) is independent of $\widetilde{X}$ which contains the hypergauge-fixing. To see this consider the transformation

$$
\delta \widetilde{\Gamma}^{\mathcal{A}}=\left(\widetilde{\Gamma}^{\mathcal{A}},-\widetilde{W}+\widetilde{X}\right) \xi+\frac{\hbar}{i}\left(\widetilde{\Gamma}^{\mathcal{A}}, \xi\right)
$$

where $\xi$ is an odd infinitesimal function satisfying the condition

$$
(\xi, \Omega)=0 .
$$

The Jacobian of (A.11) is

$$
J-1=2(-\widetilde{\Delta} \widetilde{W}+\widetilde{\Delta} \widetilde{X}) \xi+2 \frac{\hbar}{i} \widetilde{\Delta} \xi+(-\widetilde{W}+\widetilde{X}, \xi) .
$$

Summing up the total contribution from (A.11) in (A.4) one finds after use of the master equations (A.5) that what remains may be viewed as the following transformations

$$
\delta \Psi=(\Psi,-\widetilde{W}+\widetilde{X}) \xi+\frac{\hbar}{i}(\Psi, \xi), \quad \delta \widetilde{X}=2 \frac{\hbar}{i} \sigma_{\widetilde{X}} \xi
$$

However, since $Z_{T}$ is independent of $\Psi$ as was shown above only $\delta \widetilde{X}$ remains. Notice that $(\delta \widetilde{X}, \Omega)=0$ in consistency with $(\widehat{\text { A.6 }})$.

One may also notice that (A.4) is invariant under general anticanonical transformations of the form

$$
\delta \widetilde{\Gamma}^{\mathcal{A}}=\left(\widetilde{\Gamma}^{\mathcal{A}}, G\right)
$$

for any odd infinitesimal function $G$ provided $\widetilde{W}$ and $\widetilde{X}$ transform according to

$$
\delta \widetilde{W}=\sigma_{\widetilde{W}} G, \quad \delta \widetilde{X}=\sigma_{\widetilde{X}} G .
$$

This may be used to demonstrate the existence of the above formulation. Consider the abelian case

$$
\Omega_{\text {Abel }}=t_{\alpha} \mathcal{C}^{\alpha}-\pi_{\alpha}^{*} \overline{\mathcal{C}}^{\alpha}, \quad\left(\Omega_{\text {Abel }}, \Omega_{\text {Abel }}\right)=0
$$


Let the master actions here satisfy $\widetilde{W}=W$ and $\widetilde{X}=W$. (This implies that (A.6) reduces to (38).) We define then $\Omega, \widetilde{W}, \widetilde{X}$ in terms of a finite transformation of $\Omega_{\mathrm{Abel}}, W, X$ of the form (A.15)- A.16), i.e.

$$
\Omega \equiv \exp (G, \cdot) \Omega_{\text {Abel }}, \quad e^{\frac{i}{\hbar} \widetilde{W}}=e^{[\widetilde{\Delta}, G]} e^{\frac{i}{\hbar} W}, \quad e^{\frac{i}{\hbar} \widetilde{X}}=e^{[\widetilde{\Delta}, G]} e^{\frac{i}{\hbar} X} .
$$

Obviously $(\Omega, \Omega)=0$, and if $W, X$ satisfies (A.6) with $\Omega_{\text {Abel }}$ then $\widetilde{W}, \widetilde{X}$ satisfy (A.6) with $\Omega$. In this manner the non-abelian case is obtained by choosing the anticanonical generator $G$ in the following form

$$
G=\mathcal{C}_{\alpha}^{*} \Lambda_{\beta}^{\alpha} \mathcal{C}^{\beta}
$$

where the matrix $e^{\Lambda}$ changes effectively the constraint basis.

\section{Appendix B}

\section{The path integral (16) with anomalous master actions}

Consider the path integral (16) in section 2 where $T_{\alpha}$ satisfies the algebra (15) and the properties (18). According to appendix A the master actions $W$ and $X$ do no longer satisfy the master equation (间) if $P_{\alpha}^{\alpha} \neq 0$ and $Q_{\alpha}^{\alpha} \neq 0$. The appropriate generalized master equations in the latter case are

$$
\begin{aligned}
\left(i \hbar \Delta+P_{\alpha}^{\alpha}\right) e^{\frac{i}{\hbar} W}=0 & \Leftrightarrow \frac{1}{2}(W, W)=i \hbar \Delta W-i \hbar P_{\alpha}^{\alpha}, \\
\left(i \hbar \Delta+Q_{\alpha}^{\alpha}\right) e^{\frac{i}{\hbar} X}=0 & \Leftrightarrow \frac{1}{2}(X, X)=i \hbar \Delta X-i \hbar Q_{\alpha}^{\alpha} .
\end{aligned}
$$

Since a consistent theory requires $\Delta$ to be nilpotent we have from (B.1) the consistency conditions

$$
\begin{array}{lll}
\Delta\left(P_{\alpha}^{\alpha} e^{\frac{i}{\hbar} W}\right)=0 & \Leftrightarrow & \sigma_{W} P_{\alpha}^{\alpha}=0, \\
\Delta\left(Q_{\alpha}^{\alpha} e^{\frac{i}{\hbar} X}\right)=0 & \Leftrightarrow & \sigma_{X} Q_{\alpha}^{\alpha}=0 .
\end{array}
$$

The proof of the invariance under (19) as well as the independence of the gauge fixing function $\chi^{\alpha}$ given in (25)-(27) are still valid in this generalized case. We may also prove the independence of the gauge fixing action $X$ following the argument of appendix A in a reduced form. We perform then the following change of integration variables in the path integral (16):

$$
\begin{aligned}
\delta \Gamma^{A}= & \left(\Gamma^{A},-W+X\right) \mu+\frac{\hbar}{i}\left(\Gamma^{A}, \mu\right), \quad \delta \mathcal{C}^{\alpha}=\left(P_{\beta}^{\alpha}-Q_{\beta}^{\alpha}\right) \mathcal{C}^{\beta} \mu+\frac{\hbar}{i} R_{\beta}^{\alpha} \mathcal{C}^{\beta} \\
\delta \pi^{\alpha}= & \left(P_{\beta}^{\alpha}-Q_{\beta}^{\alpha}\right) \pi^{\beta} \mu+\frac{\hbar}{i} R_{\beta}^{\alpha} \pi^{\beta}+\frac{\hbar}{i} \overline{\mathcal{C}}_{\beta}\left(\chi^{\beta}, R_{\gamma}^{\alpha}\right) \mathcal{C}^{\gamma}+ \\
& +\overline{\mathcal{C}}_{\beta}\left(\chi^{\beta}, P_{\gamma}^{\alpha}-Q_{\gamma}^{\alpha}\right) \mathcal{C}^{\gamma} \mu
\end{aligned}
$$

where $\mu$ is an odd function which satisfies the condition

$$
\left(\mu, T_{\alpha}\right)=T_{\beta} R_{\alpha}^{\beta}
$$


which in turn determines $R_{\alpha}^{\beta}$. The change of integration variables (B.3) in (16) results in the following change in $X$ :

$$
\delta X=\frac{2 \hbar}{i}\left[\sigma_{X} \mu-i \hbar R_{\alpha}^{\alpha}(-1)^{\varepsilon_{\alpha}}\right]
$$

together with the following variation in $\chi^{\alpha}$ :

$$
\delta \chi^{\alpha}=\left(\chi^{\alpha},-W+X\right) \mu+\frac{\hbar}{i}\left(\chi^{\alpha}, \mu\right)
$$

which is inessential as the previous proof of $\chi$-independence remains valid.

The change $\delta X$ in $(\overline{B .5})$ is the most general one compatible with the allowed variation

$$
\delta Q_{\alpha}^{\alpha}=\frac{2 \hbar}{i}\left[\sigma_{X} R_{\alpha}^{\alpha}(-1)^{\varepsilon_{\alpha}}+\left(Q_{\alpha}^{\alpha}, \mu\right)\right]
$$

in the modified equation for $X$ given in (B.1). This variation is the trace of the matrix variation

$$
\delta Q_{\beta}^{\alpha}=\frac{2 \hbar}{i}\left[\sigma_{X} R_{\beta}^{\alpha}(-1)^{\varepsilon_{\alpha}}+\left(Q_{\beta}^{\alpha}, \mu\right)+Q_{\gamma}^{\alpha} R_{\beta}^{\gamma}-R_{\gamma}^{\alpha} Q_{\beta}^{\gamma}+i \hbar S_{\gamma \beta}^{\alpha \gamma}\right]
$$

where in turn $S_{\gamma \beta}^{\alpha \gamma}$ is determined by the relation

$$
\begin{aligned}
& \left(R_{\alpha}^{\gamma}, T_{\beta}\right)-\left(R_{\beta}^{\gamma}, T_{\alpha}\right)(-1)^{\left(\varepsilon_{\alpha}+1\right)\left(\varepsilon_{\beta}+1\right)}+(-1)^{\varepsilon_{\gamma}}\left(\mu, U_{\alpha \beta}^{\gamma}\right)- \\
& -R_{\delta}^{\gamma} U_{\alpha \beta}^{\delta}+U_{\alpha \delta}^{\gamma} R_{\beta}^{\delta}-U_{\beta \delta}^{\gamma} R_{\alpha}^{\delta}(-1)^{\left(\varepsilon_{\alpha}+1\right)\left(\varepsilon_{\beta}+1\right)}+T_{\delta} S_{\alpha \beta}^{\delta \gamma}(-1)^{\varepsilon_{\gamma}}=0,
\end{aligned}
$$

which is a compatibility condition to (B.4). In terms of $\delta Q_{\beta}^{\alpha}$ we have

$$
\left(\delta X, T_{\alpha}\right)=T_{\beta} \delta Q_{\alpha}^{\beta} .
$$

The main part of these formulas may be derived from (A.11)-A.14 with the ansatz

$$
\xi=\mu+\mathcal{C}_{\alpha}^{*} R_{\beta}^{\alpha} \mathcal{C}^{\beta}+\frac{1}{4} \mathcal{C}_{\alpha}^{*} \mathcal{C}_{\beta}^{*} S_{\delta \gamma}^{\beta \alpha} \mathcal{C}^{\gamma} \mathcal{C}^{\delta}(-1)^{\varepsilon_{\delta}}+\ldots
$$

\section{Appendix C}

\section{Proof of formula (33)}

Inserting the ansatz (30) into 29 ) one finds to the zeroth order in $\Phi^{\alpha}$ the condition

$$
\left(\Theta^{\alpha}, \Theta^{\beta}\right) \equiv E^{\alpha \beta}=-(-1)^{\varepsilon_{\gamma}\left(1+\varepsilon_{\alpha}\right)} X_{\gamma}^{\alpha} \omega^{\gamma \rho} X_{\rho}^{\beta}
$$

for the first order coefficients $X_{\beta}^{\alpha}(\Gamma)$ in (30). This implies that

$$
E_{\alpha \beta}=-(-1)^{\varepsilon_{\beta}\left(1+\varepsilon_{\gamma}\right)}\left(X^{-1}\right)_{\alpha}^{\rho} \omega_{\rho \gamma}\left(X^{-1}\right)_{\beta}^{\gamma},
$$

where $\left(X^{-1}\right)_{\alpha}^{\rho}$ is the inverse to $X_{\beta}^{\alpha}$ in the sense

$$
\left(X^{-1}\right)_{\alpha}^{\gamma} X_{\gamma}^{\beta}=X_{\alpha}^{\gamma}\left(X^{-1}\right)_{\gamma}^{\beta}=\delta_{\alpha}^{\beta} .
$$


For the gauge invariant functions $\bar{F}$ and $\bar{G}$ in (33) we have to the first order in $\Phi$

$$
\bar{F}=F(\Gamma)+\Phi^{\alpha} Y_{\alpha}(F)+O\left(\Phi^{2}\right), \quad \bar{G}=G(\Gamma)+\Phi^{\alpha} Y_{\alpha}(G)+O\left(\Phi^{2}\right),
$$

where

$$
\begin{aligned}
Y_{\alpha}(G) & =-(-1)^{\varepsilon_{\alpha}\left(1+\varepsilon_{G}\right)}\left(G, \Theta^{\gamma}\right)\left(X^{-1}\right)_{\gamma}^{\beta} \omega_{\beta \alpha}= \\
& =-(-1)^{\varepsilon_{\gamma}\left(1+\varepsilon_{\beta}\right)} \omega_{\alpha \beta}\left(X^{-1}\right)_{\gamma}^{\beta}\left(\Theta^{\gamma}, G\right) .
\end{aligned}
$$

These expressions and (C.2) imply now

$$
\begin{aligned}
& \left.(\bar{F}, \bar{G})_{\mathrm{ext}}\right|_{\Phi=0}=(F, G)+(-1)^{\varepsilon_{\alpha}\left(1+\varepsilon_{F}\right)} Y_{\alpha}(F) \omega^{\alpha \beta} Y_{\beta}(G)= \\
& =(F, G)-\left(F, \Theta^{\alpha}\right) E_{\alpha \beta}\left(\Theta^{\beta}, G\right) \equiv(F, G)_{(\mathcal{D})} .
\end{aligned}
$$

\section{Equation for $\Theta_{\alpha}^{*}$ in $(57)$}

In parallel to (30), if one inserts the ansatz (57) into eq. (56) one gets to the zeroth order in $\Phi^{\alpha}$ the equations

$$
\begin{gathered}
\left(\Theta^{\alpha}, \Theta_{\beta}^{*}\right)+(-1)^{\varepsilon_{\mu}\left(1+\varepsilon_{\alpha}\right)} X_{\mu}{ }^{\alpha} \omega^{\mu \nu} X_{\nu \beta}^{*}=\delta_{\beta}^{\alpha}, \\
\left(\Theta_{\alpha}^{*}, \Theta_{\beta}^{*}\right)+(-1)^{\varepsilon_{\mu} \varepsilon_{\alpha}} X_{\mu \alpha}^{*} \omega^{\mu \nu} X_{\nu \beta}^{*}=0 .
\end{gathered}
$$

Solving (C.7) for $X_{\alpha \beta}^{*}$ one finds

$$
X_{\alpha \beta}^{*}=-\left(\delta_{\beta}^{\gamma}+\left(\Theta_{\beta}^{*}, \Theta^{\gamma}\right)\right)\left(X^{-1}\right)_{\gamma}^{\delta} \omega_{\delta \alpha}(-1)^{\varepsilon_{\alpha} \varepsilon_{\beta}},
$$

which when inserted into (C.8) results in the following equation for $\Theta_{\alpha}^{*}$ :

$$
\left(\Theta_{\alpha}^{*}, \Theta_{\beta}^{*}\right)_{(\mathcal{D})}=-E_{\alpha \beta}-\left(\Theta_{\alpha}^{*}, \Theta^{\gamma}\right) E_{\gamma \beta}+E_{\alpha \gamma}\left(\Theta^{\gamma}, \Theta_{\beta}^{*}\right) .
$$

The solution is of the form

$$
\Theta_{\alpha}^{*}=\frac{1}{2} \Theta^{\gamma} E_{\gamma \alpha}+O\left(\Theta^{2}\right)
$$

To confirm the existence of $T_{\alpha}^{*}$ satisfying (56) we notice that for abelian second class constraints $\theta^{\alpha}(\Gamma)=0$ satisfying $\left(\theta^{\alpha}, \theta^{\beta}\right)=-\omega^{\alpha \beta}$ we have the following explicit conversion formula for corresponding abelian first class constraints $t^{\alpha}$ and $t_{\alpha}^{*}$ :

$$
t^{\alpha}=\theta^{\alpha}+\Phi^{\alpha}, \quad t_{\alpha}^{*}=-\frac{1}{2}\left(\theta^{\gamma}-\Phi^{\gamma}\right) \omega_{\gamma \alpha}
$$

The general abelian functions $T^{\alpha}$ and $T_{\alpha}^{*}$ are then obtained from $t^{\alpha}$ and $t_{\alpha}^{*}$ through the formula

$$
T^{\alpha}=\exp (G, \cdot)_{\operatorname{ext}} t^{\alpha}, \quad T_{\alpha}^{*}=\exp (G, \cdot)_{\operatorname{ext}} t_{\alpha}^{*}
$$

where $G(\Gamma, \Phi)$ is an odd function. 


\section{References}

[1] I.A. Batalin and G.A. Vilkovisky, Phys. Lett. B102, 27 (1981);ibid B120, 166 (1983); Phys. Rev. D28, 2567 (1983); J. Math. Phys. 26, 172 (1985)

[2] J. Zinn-Justin, Renormalization of gauge theories, in Trends in Elementary Particle Theory, Lecture Notes in Physics (eds. Rollnik and Dietz), vol 37, Springer-Verlag, Berlin, 1975.

[3] R. Kallosh, JETP Lett. 26, 575 (1977)

[4] B. de Wit and J. van Holten, Phys. Lett. B79, 389 (1979)

[5] D. V. Volkov, V. A. Soroka, A. I. Pashnev, and V. I. Tkach, JETP Lett. 44, 55 (1986)

[6] M. Henneaux, Nucl. Phys. (Proc.Suppl.) 18A, 47 (1990)

[7] E. Witten, Modern Phys. Lett. A5, 487 (1990)

[8] O. M. Khudaverdyan, J. Math. Phys.. 32, 1934 (1991);

O. M. Khudaverdyan and A. Nersessian, Mod. Phys. Lett. A8, 2377 (1993);

[9] A. Schwarz, Comm. Math. Phys.. 155, 249 (1993); ibid 158, 373 (1993);

H. Hata and B. Zweibach, Ann. Phys. (N. Y.) 229, 177 (1994);

J. Alfaro and P. H. Damgaard, Nucl. Phys. B455, 409 (1995)

[10] I.A. Batalin and I.V. Tyutin, Int. J. Mod. Phys. A8, 2333 (1993).

[11] I.A. Batalin and I.V. Tyutin, Mod. Phys. Lett. A8, 3673 (1993); ibid A9, 1707 (1994)

[12] I.A. Batalin and I.V. Tyutin, Int. J. Mod. Phys. A9, 517 (1994);

F. Akman, q-alg/9506027; I. A. Batalin, K. Bering, and P. H. Damgaard, Phys. Lett. B389, 673 (1996)

[13] I.A. Batalin and I.V. Tyutin, Amer. Math. Soc. Transl. 177, 23 (1996)

[14] I. A. Batalin, R. Marnelius and A. M. Semikhatov, Nucl. Phys. B446, 249 (1995)

[15] I. A. Batalin and R. Marnelius, Nucl. Phys. B465, 521 (1996)

[16] I. A. Batalin and E. S. Fradkin, Nucl. Phys. B279, 514 (1987);

Phys. Lett. B180, 157 (1986); ibid B236, 528 (1990);

I. A. Batalin, E. S. Fradkin, and T. E. Fradkina, Nucl. Phys. B314, 158 (1989); ibid B323, 734 (1989)

I. A. Batalin and I. V. Tyutin, Int. J. Mod. Phys. A6, 3599 (1991)

[17] I. A. Batalin, E. S. Fradkin, and T. E. Fradkina, Nucl. Phys. B332, 723 (1990)

[18] L. Faddeev and S. Shatashvili, Phys. Lett. 167B, 225 (1986)

[19] E. S. Fradkin and V. Ya. Linetskii, Nucl. Phys. B431, 569 (1994);

ibid B444, 577 (1995) 